\title{
Portfolio optimization of credit swap under funding costs
}

\author{
Lijun Bo
}

Received: 27 January 2017 / Accepted: 17 October 2017 / Published online: 04 December 2017 (C) The Author(s). 2017 Open Access This article is distributed under the terms of the Creative Commons Attribution 4.0 International License (http://creativecommons.org/licenses/by/4.0/), which permits unrestricted use, distribution, and reproduction in any medium, provided you give appropriate credit to the original author(s) and the source, provide a link to the Creative Commons license, and indicate if changes were made.

\begin{abstract}
We develop a dynamic optimization framework to assess the impact of funding costs on credit swap investments. A defaultable investor can purchase CDS upfronts, borrow at a rate depending on her credit quality, and invest in the money market account. By viewing the concave drift of the wealth process as a continuous function of admissible strategies, we characterize the optimal strategy in terms of a relation between a critical borrowing threshold and two solutions of a suitably chosen system of first order conditions. Contagion effects between risky investor and reference entity make the optimal strategy coupled with the value function of the control problem. Using the dynamic programming principle, we show that the latter can be recovered as the solution of a nonlinear HJB equation whose coeffcients admit singular growth. By means of a truncation technique relying on the locally Lipschitzcontinuity of the optimal strategy, we establish existence and uniqueness of a global solution to the HJB equation.
\end{abstract}

\section{Introduction}

The vast majority of literature studying portfolio allocation strategies across fixedincome securities has focused on a single source of defaults. Kraft and Steffensen (2005) analyze optimal investment in an asset defaulting when an economic state process falls below a given threshold. Bielecki and Jang (2006) derive optimal investment strategies for an investor allocating her wealth among a defaultable bond, risk-free account and stock, assuming constant default intensity. Bo et al. (2010)

\footnotetext{
L. Bo $(\bowtie)$

School of Mathematics and Statistics, Xidian University, Xian 710071, China

e-mail: lijunbo@ustc.edu.cn

L. Bo

School of Mathematical Sciences, University of Science and Technology of China, Hefei, Anhui Province 230026, China
} 
consider a logarithmic investor maximizing utility from consumption in market model consisting of a defaultable perpetual bond, default-free stock, and money market account. Capponi and Figueroa-López (2014) study an economy consisting of a stock and of a defaultable bond whose price processes are modulated by an observable Markov chain. Jiao and Pham (2011) decompose a global optimal investment problem into sub-control problems defined in a progressively enlarged filtration in an economy consisting of a risk-free bond and a stock subject to counterparty risk. Few other studies have considered portfolio frameworks consisting of multiple defaultable securities. Using a static model, Giesecke et al. (2014) study the portfolio selection problem of an investor who maximizes the mark-to-market value of a fixed income portfolio of credit default swaps. Bielecki et al. (2008) study how dynamic investment in credit default swaps may be used to replicate defaultable contingent claims.

When financing investment strategies, the investor is typically charged a higher rate for borrowing than for lending. In the absence of default risk, the divergence between borrowing and lending rates has been analyzed both in the context of claim valuation by Korn (1995), and of hedging in incomplete markets by Cvitanić and Karatzas (1993). We also refer to El et al. (1997) who study the super-hedging price of contingent claims under asymmetry of borrowing and lending interest rates via nonlinear backward stochastic differential equations. When financing credit investment strategies referencing high-risk names, the investor would need to borrow at her own refinancing rate rather than at the repo rate. This rate for unsecured borrowing depends on the credit quality of the investor who will be charged a spread reflecting her default likelihood as perceived by the market. Moreover, contagion effects generated from the default risk of the credit sensitive security introduce complex dependence patterns between the borrowing spreads and the mark-to-market price of the CDS security. We also remark that the recent crisis has underscored the importance of including funding costs in optimal replication and investment strategies so to account for liquidity and counterparty risk, see also Crépey (2015) and Bielecki and Rutkowski (2015).

The main goal of the paper is to rigorously analyze the impact of borrowing costs incurred by a defaultable investor on her optimal allocation strategy. We construct a dynamic optimization framework where a defaultable investor can buy CDS upfronts, borrow at a premium over the risk-free rate, and invest in a money market account at the risk-free rate. The choice of upfront CDSs reflects regulatory market practices following the Big Bang Protocol (ISDA News Release 2009), and requiring distressed credits to trade with an upfront fee plus a running fixed coupon depending on the credit quality of the reference entity. The borrowing rate of the investor accounts both for her credit quality and for feedback effects generated by default of the reference entity. The latter are modeled using a contagion credit risk model with interacting default intensities, see also Jarrow and Yu (2001) for more explicit examples and Frey and Backhaus (2008) for a theoretical illustration. The investor maximizes her power utility from terminal wealth until the earlier of either her default time or the investment horizon.

Building on (Kraft and Steffensen 2005), we use the dynamic programming principle to characterize the HJB equations corresponding to the different default states. When the investor defaults, the solution to the HJB equation is specified 
as a boundary condition. Due to borrowing costs incurred by the investor when financing her purchases, the wealth process admits a concave drift, which is not differentiable with respect to the investment strategy. This prohibits the use of firstorder conditions for analyzing the optimal strategy. Moreover, because of defaults and contagion effects, we have to deal with the presence of jumps both in the price and in the wealth dynamics, and with concave drift appearing only in the wealth but not in the price dynamics. This leads us to apply a different method which considers the concave drift as a continuous function of the admissible strategy $\psi$, differentiable everywhere except at a critical point $\ell$. Such a critical point defines two regions where the optimal admissible control may lie. More specifically, we decompose the component $H(\psi)$ of the HJB equation yielding the optimal strategy as $H(\psi)=\mathbf{1}_{\psi \leq \ell} H_{1}(\psi)+\mathbf{1}_{\psi>\ell} H_{2}(\psi)$, where $H_{1}(\psi)$ and $H_{2}(\psi)$ are two continuously differentiable functions. We analyze the solutions of the system of first order conditions associated with $H_{1}(\psi)$ and $H_{2}(\psi)$, both seen as functions on their entire domains. The similar method has been proposed in Bo and Capponi (2017) for studying the fixed-income portfolio optimization with borrowing costs. We then characterize the optimal strategy in terms of a relation between the critical point and the previously established solutions. Default contagion makes the optimal strategy coupled with the value function, which is recovered as the solution of the corresponding nonlinear ordinary differential HJB equation. Moreover, we can prove that the coefficients of this equation admit a singular growth. By means of a delicate analysis, we establish the existence and uniqueness of a global solution to the HJB equation in two main steps. First, we prove the locally Lipschitz-continuity of the optimal strategy when seen as a function of the value function (see Lemma 8 for details). We then show that if a solution to the HJB equation exists, then it must have a strictly positive uniform lower bound. This allows us to develop a novel truncation technique to remove the singularity in the HJB equation, and subsequently prove the existence and uniqueness of a global solution to the HJB equation.

The rest of the paper is organized as follows. "The model" section develops the default and market model. "Price dynamics and borrowing rates" section provides the dynamics of the CDS price process and analyzes borrowing rates. "Dynamic investment problem" section formulates the dynamic investment problem. "Optimal feedback strategy" section studies optimal investment strategies, while "Solvability of HJB equations" section establishes existence and uniqueness of solutions for the HJB equations. Some technical proofs are delegated to the Appendix.

\section{The model}

We describe the default model in "Default contagion model" section and the market model in "The market model" section. Throughout the paper, we refer to " 1 " as the reference entity of the CDS, and to " $I$ " as the investor.

\section{Default contagion model}

The default state is described by a 2-dimensional default indicator process $\mathbf{H}(t)=$ $\left(H_{1}(t), H_{I}(t)\right), t \geq 0$, supported by a filtered probability space $(\Omega, \mathcal{G}, \mathbb{Q})$. Here $\mathbb{Q}$ 
denotes the risk-neutral probability measure. Further, $H_{i}(t)=1$ if $i$ has defaulted by time $t$ and $H_{i}(t)=0$ otherwise, $i \in\{1, I\}$. This means that the state space of the default indicator process $\mathbf{H}=(\mathbf{H}(t) ; t \geq 0)$ is given by $\mathcal{S}:=\{0,1\}^{2}$. The default time of the $i$-th name is defined by $\tau_{i}=\inf \left\{t \geq 0 ; H_{i}(t)=1\right\}$ for $i \in\{1, I\}$. Then it holds that $H_{i}(t)=\mathbf{1}_{\left\{\tau_{i} \leq t\right\}}$ for $t \geq 0$.

We model default contagion through a Markovian model with interacting intensities. The default indicator process $\mathbf{H}$ is assumed to follow a continuous time Markov chain on $\mathcal{S}$, where $\mathbf{H}(t)$ transits to the neighbouring state $\mathbf{H}^{1}(t):=\left(1-H_{1}(t), H_{I}(t)\right)$ at rate $\mathbf{1}_{\left\{H_{1}(t)=0\right\}} h_{1, \mathbf{H}(t)}(t)$, and to the neighbouring state $\mathbf{H}^{I}(t):=\left(H_{1}(t), 1-H_{I}(t)\right)$ at rate $\mathbf{1}_{\left\{H_{I}(t)=0\right\}} h_{I, \mathbf{H}(t)}(t)$. Here for $i \in\{1, I\}, h_{i, \mathbf{z}}(t)$ is a positive bounded measurable function with a strictly positive lower bound. We also refer the reader to Frey and Backhaus (2008) for explicit probabilistic models under this setup. Hence, $\mathbf{H}$ admits the following $\mathbb{Q}$-infinitesimal generator given by

$$
\mathcal{A} g_{\mathbf{z}}(t)=\sum_{j \in\{1, I\}}\left(1-z_{j}\right) h_{j, \mathbf{z}}(t)\left[g_{\mathbf{z}^{j}}(t)-g_{\mathbf{z}}(t)\right],
$$

where $g_{\mathbf{z}}(t)$ is an arbitrary measurable function and the default state vectors

$$
\mathbf{z}^{1}:=\left(1-z_{1}, z_{I}\right), \quad \mathbf{z}^{I}:=\left(z_{1}, 1-z_{I}\right), \quad z \in \mathcal{S} .
$$

The market filtration is given by $\mathcal{G}_{t}=\sigma(\mathbf{H}(s) ; s \leq t)$. We take the right continuous version of $\mathcal{G}_{t}$, i.e. $\mathcal{G}_{t}:=\cap_{\epsilon>0} \mathcal{G}_{t+\epsilon}$ (see also Belanger et al. (2004)). Using the Dynkin's formula by choosing $g_{\mathbf{z}}(t)=z_{i}$, it follows that

$$
\xi_{i}(t):=H_{i}(t)-\int_{0}^{t}\left(1-H_{i}(s)\right) h_{i, \mathbf{H}(s)}(s) d s, \quad t \geq 0
$$

is a martingale. At time 0 , we place ourselves in the scenario where all names do not default, i.e. $\mathbf{H}(0)=\mathbf{0}$, where $\mathbf{0}$ is a two dimensional vector consisting of zero entries.

\section{The market model}

Consider a financial market consisting of a lending account, a borrowing account and an upfront CDS.

- Lending account. The investor lends at a constant risk-free rate $r>0$. The time- $t$ price of one share of his lending account is $B_{t}=e^{r t}$ for $t \geq 0$.

- Borrowing account. We proxy the borrowing costs incurred by investor with her bond credit spreads. We also refer to Chen (2010) for a dynamic model addressing the credit spread puzzle along with its relation to borrowing costs. In our model, credit spreads account both for the default risk of the investor and for contagion effects induced by the reference entity underlying the CDS. More specifically, the borrowing account of the investor is given by $\bar{B}_{t}=e^{\int_{0}^{t} r_{\mathbf{H}(s)}(s) d s}$, 
$t \geq 0$, where $r_{\mathbf{H}(t)}(t)$ denotes the default state dependent borrowing rate. The latter will be analyzed in detail in "Borrowing rates" section.

- Upfront CDS. In a traditional running CDS contract a spread is paid throughout the life of the contract. The Big Bang Protocol introduced by ISDA News Release (2009) requires the premium leg to perform one of the following actions:

1. Make one single payment at the initiation of the CDS contract for protection until maturity.

2. Make one upfront payment plus pay a running premium until the earlier of a credit event or maturity. The running premium is set much lower than it would be under the traditional method.

Upfront CDSs greatly reduce the counterparty exposure of the protection seller to the protection buyer. Currently, most CDS trades follow the upfront mechanism, especially for distressed credits. For a given loss rate $L>0$ paid at default of the reference entity " 1 ", we choose the contractual running spread premium $v>0$ so that it satisfies the following upfront condition:

(A1) $L \inf _{t \geq 0} h_{1,00}(t)-v>0$.

The above condition states that the running premium $v$ is decided in such a way that the minimum expected loss rate paid throughout the life of the transaction $L$ inf $_{t \geq 0} h_{1,00}(t)$ is always higher than the spread premium $v$. Consequently, a positive money amount would have to be posted by the protection buyer at the inception of the trade. Let $T_{1}>0$ be the maturity of the CDS. Then, the dividend process is given by, for $t \in\left[0, T_{1}\right]$,

$$
D_{t}:=L H_{1}(t)-v \int_{0}^{t}\left(1-H_{1}(s)\right) d s, \quad D_{0}=0 \text {. }
$$

The ex-dividend price of the CDS at time- $t$ is given by

$$
C_{t}=\left(1-H_{1}(t)\right) B_{t} \mathbb{E}_{t}\left[\int_{t}^{T} B_{u}^{-1} d D_{u}\right] .
$$

Here $\mathbb{E}_{t}$ denotes the expectation operator conditional on $\mathcal{G}_{t}$ under $\mathbb{Q}$. Throughout the paper, we consider the following contagion condition.

(A2) The default intensities satisfy

$$
h_{1,01}(t)>h_{1,00}(t) \text { for } t \in\left[0, T_{1}\right] \text {, and } h_{I, 10}(t)>h_{I, 00}(t) \text { for } t \in[0, T],
$$

where $T_{1}>0$ is the maturity of the CDS, and $T \in\left(0, T_{1}\right)$ denotes the terminal time of the borrowing account. Such a condition captures the self-exciting feature of default events, empirically tested in several studies, see, for instance, Azizpour et al. (2017) for the case of U.S. corporate defaults. 


\section{Price dynamics and borrowing rates}

We price CDS in "CDS pricing" section and analyze the investor's borrowing costs in "Borrowing rates" section.

\section{CDS pricing}

From (5), the price process of the CDS can be rewritten as, for $t \in\left[0, T_{1}\right]$,

$$
C_{t}=\left(1-H_{1}(t)\right) \Gamma_{\mathbf{H}(t)}(t) .
$$

Here $\Gamma_{\mathbf{z}}(t),(t, \mathbf{z}) \in\left[0, T_{1}\right] \times \mathcal{S}$, is the price function of the CDS contract given by

$$
\Gamma_{\mathbf{z}}(t):=L \Gamma_{\mathbf{z}}^{(1)}(t)-v \Gamma_{\mathbf{z}}^{(2)}(t),
$$

where for $(t, \mathbf{z}) \in\left[0, T_{1}\right] \times \mathcal{S}$,

$$
\begin{aligned}
\Gamma_{\mathbf{z}}^{(1)}(t) & :=\mathbb{E}_{t, \mathbf{z}}\left[H_{1}\left(T_{1}\right) e^{\left.-\int_{t}^{T_{1}} r\left(1-H_{1}(u)\right) d u\right],}\right. \\
\Gamma_{\mathbf{z}}^{(2)}(t) & :=\mathbb{E}_{t, \mathbf{z}}\left[\int_{t}^{T_{1}} e^{-\int_{t}^{u} r d s}\left(1-H_{1}(u)\right) d u\right] .
\end{aligned}
$$

Using Feymann-Kac formula, $\Gamma_{\mathbf{z}}^{(1)}(t)$ and $\Gamma_{\mathbf{z}}^{(2)}(t)$ solve respectively

$$
\left\{\begin{array}{l}
\left(\frac{\partial}{\partial t}+\mathcal{A}\right) \Gamma_{\mathbf{z}}^{(1)}(t)=r\left(1-z_{1}\right) \Gamma_{\mathbf{z}}^{(1)}(t), \quad \Gamma_{\mathbf{z}}^{(1)}\left(T_{1}\right)=z_{1}, \\
\left(\frac{\partial}{\partial t}+\mathcal{A}\right) \Gamma_{\mathbf{z}}^{(2)}(t)+\left(1-z_{1}\right)=r \Gamma_{\mathbf{z}}^{(2)}(t), \Gamma_{\mathbf{z}}^{(2)}\left(T_{1}\right)=0
\end{array}\right.
$$

where the operator $\mathcal{A}$ is given by (1). It is not difficult to get that

Lemma 1 For $(t, \mathbf{z}) \in\left[0, T_{1}\right] \times \mathcal{S}$, the CDS price function $\Gamma_{\mathbf{z}}(t)$ has the closedform representations:

$$
\left\{\begin{array}{l}
\Gamma_{10}(t)=\Gamma_{11}(t)=L ; \\
\Gamma_{01}(t)=\int_{t}^{T_{1}}\left(L h_{1,01}(s)-v\right) e^{-\int_{t}^{s}\left(r+h_{1,01}(u)\right) d u} d s ; \\
\Gamma_{00}(t)=\int_{t}^{T_{1}}\left(L h_{1,00}(s)-v+h_{I, 00}(s) \Gamma_{01}(s)\right) e^{-\int_{t}^{s}\left(r+h_{1,00}(u)+h_{I, 00}(u)\right) d u} d s .
\end{array}\right.
$$

Also for all $t \in\left[0, T_{1}\right), \Gamma_{01}(t)>\Gamma_{00}(t)$, and $\Gamma_{01}\left(T_{1}\right)=\Gamma_{00}\left(T_{1}\right)=0$.

Lemma 2 The $\mathbb{Q}$-dynamics of the CDS price process is given by, for $t \in\left[0, T_{1}\right)$,

$$
\begin{aligned}
d C_{t}= & \left(1-H_{1}(t)\right)\left[r C_{t}+\left(v-h_{1, \mathbf{H}(t)}(t) L\right)\right] d t-C_{t-} d \xi_{1}(t) \\
& +\left(1-H_{1}(t-)\right)\left[\Gamma_{\mathbf{H}^{I}(t-)}(t)-C_{t-}\right] d \xi_{I}(t) .
\end{aligned}
$$

The investor wishes to optimize his expected utility under the measure that describes the actual distribution of risk factors governing the market value. We provide a formula which allows for identifying the historical measure $\mathbb{P}$ from the risk neutral measure $\mathbb{Q}$ under which the price processes are observed. Let $\lambda_{i, \mathbf{z}}(t)$ be a bounded measurable function defined on $(t, \mathbf{z}) \in \mathbb{R}_{+} \times \mathcal{S}$, which takes values on 
$(-1, \infty)$, where $i \in\{1, I\}$. Assume that the process $X=\left(X_{t} ; t \geq 0\right)$ satisfies the following SDE given by

$$
\frac{d X_{t}}{X_{t-}}=\sum_{i \in\{1, I\}} \lambda_{i, \mathbf{H}(t-)}(t) d \xi_{i}(t), \quad X_{0}=1,
$$

where the $\mathbb{Q}$-default martingale process $\xi_{i}$ is defined by (3). Define a new probability measure $\mathbb{P} \ll \mathbb{Q}$ on $\mathcal{G}_{T}$ by $d \mathbb{P}=X_{T} d \mathbb{Q}$. Then, for $i \in\{1, I\}$,

$$
\xi_{i}^{\mathbb{P}}(t):=H_{i}(t)-\int_{0}^{t}\left(1-H_{i}(s)\right) h_{i, \mathbf{H}(s)}^{\mathbb{P}}(s) d s, \quad t \in[0, T]
$$

is a $\mathbb{P}$-martingale, where the relation between the $\mathbb{P}$-default intensity of the $i$-th default indicator $H_{i}(t)$ and its $\mathbb{Q}$-default intensity is given by

$$
h_{i, \mathbf{z}}^{\mathbb{P}}(t)=h_{i, \mathbf{z}}(t)\left(1+\lambda_{i, \mathbf{z}}(t)\right), \quad(t, \mathbf{z}) \in[0, T] \times \mathcal{S} .
$$

Then from Lemma 2 , it follows that the $\mathbb{P}$-dynamics of the CDS price process is given by for $t \in\left[0, T_{1}\right)$,

$$
\begin{aligned}
d C_{t}= & \left(1-H_{1}(t)\right)\left[r C_{t}+\left(v-h_{1, \mathbf{H}(t)}(t) L\right)\right] d t \\
& -C_{t-} d\left\{\xi_{1}^{\mathbb{P}}(t)+\int_{0}^{t}\left(1-H_{1}(s)\right) \lambda_{1, \mathbf{H}(s)}(s) h_{1, \mathbf{H}(s)}(s) d s\right\} \\
& +\left(1-H_{1}(t-)\right)\left[\Gamma_{\mathbf{H}^{I}(t-)}(t)-C_{t-}\right] d \\
& \times\left\{\xi_{I}^{\mathbb{P}}(t)+\int_{0}^{t}\left(1-H_{I}(s)\right) \lambda_{I, \mathbf{H}(s)}(s) h_{I, \mathbf{H}(s)}(s) d s\right\} .
\end{aligned}
$$

\section{Borrowing rates}

The default state dependent borrowing rate function of the investor $r_{\mathbf{z}}(t),(t, \mathbf{z}) \in$ $[0, T] \times \mathcal{S}$, is given by the yield spread of a zero coupon bond written by the investor and expiring at $T$. For $(t, \mathbf{z}) \in[0, T] \times \mathcal{S}$, we use $\Phi_{\mathbf{z}}(t, T)$ to denote the time- $t$ price function of the investor bond with maturity $T>t$, given by

$$
\Phi_{\mathbf{z}}(t, T)=\mathbb{E}_{t, \mathbf{z}}\left[e^{-\int_{t}^{T} r d s}\left(1-H_{I}(T)\right)\right] .
$$

Following Duffie and Singleton (2003), Section 5.3, define the default state dependent borrowing rate of the investor when the investor does not default at time $t$ as, for $z_{1} \in\{0,1\}$

$$
r_{z_{1} 0}(t):=\frac{1}{T-t} \log \left(\frac{1}{\Phi_{z_{1} 0}(t, T)}\right), \quad t \in[0, T) .
$$

When time to maturity approaches zero, we define $r_{z_{1} 0}(T)$ with $z_{1} \in\{0,1\}$ as the limiting values, i.e.

$$
r_{z_{1} 0}(T):=\lim _{t \uparrow T} \frac{1}{T-t} \log \left(\frac{1}{\Phi_{z_{1} 0}(t, T)}\right) .
$$

However, when the investor has defaulted at time $t$, he can not invest the borrowing account anymore, and hence the borrowing rates of the investor $r_{z_{1} 1}(t)$ with 
$z_{1} \in\{0,1\}$ are not needed. In order to be consistent mathematically, we set $r_{z_{1}}(t)=$ 0 for all $t \in[0, T]$.

We next develop an explicit representation of $\Phi_{\mathbf{z}}(t, T)$. Recall the operator $\mathcal{A}$ given by (1). It follows from (14) that on $(t, \mathbf{z}) \in[0, T) \times \mathcal{S}$,

$$
\left(\frac{\partial}{\partial t}+\mathcal{A}\right) \Phi_{\mathbf{z}}(t, T)=r \Phi_{\mathbf{z}}(t, T)
$$

and $\Phi_{\mathbf{z}}(T, T)=1-z_{I}$. Then for $t \in[0, T]$,

$$
\left\{\begin{aligned}
\Phi_{01}(t, T) & =\Phi_{11}(t, T)=0 ; \\
\Phi_{10}(t, T) & =e^{-\int_{t}^{T}\left(r+h_{I, 10}(u)\right) d u} ; \\
\Phi_{00}(t, T) & =e^{-\int_{t}^{T}\left(r+h_{1,00}(u)+h_{I, 00}(u)\right) d u} \\
& +\Phi_{10}(t, T) \int_{t}^{T} h_{1,00}(s) e^{-\int_{t}^{s} h_{1,00}(u) d u} e^{\int_{t}^{s}\left(h_{I, 10}(u)-h_{I, 00}(u)\right) d u} d s .
\end{aligned}\right.
$$

Also for all $t \in\left[0, T_{1}\right), \Phi_{10}(t, T)<\Phi_{00}(t, T)$, and $\Phi_{10}(T, T)=\Phi_{00}(T, T)=1$. In the following, we analyze the borrowing rate functions $r_{z_{1} 0}(t)$ defined by (15). Using (15), we have

$$
r_{10}(t)=\frac{1}{T-t} \log \left(\frac{1}{\Phi_{10}(t, T)}\right), r_{00}(t)=\frac{1}{T-t} \log \left(\frac{1}{\Phi_{00}(t, T)}\right) .
$$

When time to maturity approaches zero, $r_{10}(T)$ and $r_{00}(T)$ are given by the limiting values (16). The exact expressions are given in the following lemma whose proof is reported in the Appendix.

Lemma 3 It holds that $r_{10}(T)=r+h_{I, 10}(T)$ and $r_{00}(T)=r+h_{I, 00}(T)$. Moreover $r+\underline{m}_{I, 10} \leq r_{10}(t) \leq r+\bar{m}_{I, 10}$ and $r+\underline{m}_{I, 00} \leq r_{00}(t)<r+\bar{m}_{1,00}+\bar{m}_{I, 00}$ for all $t \in[0, T]$. Here $\underline{m}_{i, \mathbf{z}}:=\inf _{t \in[0, T]} h_{i, \mathbf{z}}(t)$ and $\bar{m}_{i, \mathbf{z}}:=\sup _{t \in[0, T]} h_{i, \mathbf{z}}(t)$.

\section{Dynamic investment problem}

We derive the wealth dynamics in "Wealth process" section and formulate the optimal control problem in "The optimal control problem" section.

\section{Wealth process}

We consider an investor who wants to maximize her power utility from terminal wealth at time $T$ by dynamically allocating her wealth across a CDS, financing her purchases using the borrowing account, and lending using the risk-free bank account. The investor has neither intermediate consumption nor capital income to support her purchase of financial assets. Denote by $\phi(t)$ the number of shares of the CDS that the investor buys $(\phi(t)>0)$ or sells $(\phi(t)<0)$ at time $t$. Similarly, $\phi_{0}^{l}(t)$ represents the number of shares invested in the lending account, and $\phi^{b}(t)$ the number of shares invested in her borrowing account. By definition $\phi_{0}^{l}(t) \geq 0$, while $\phi^{b}(t) \leq 0$. We 
assume that simultaneous borrowing and lending is not efficient, i.e., $\phi^{l}(t) \phi^{b}(t)=0$. We remark that similar assumptions have been made by Bielecki and Rutkowski (2015) and, by Mercurio (2015). The wealth process of a portfolio $\phi=\left(\phi, \phi^{l}, \phi^{b}\right)$ equals

$$
V_{t}^{\phi}=\phi(t) C_{t}+\phi^{l}(t) B_{t}+\phi^{b}(t) \bar{B}_{t}
$$

As usual, we require the portfolio process $\phi$ to be $\mathbb{G}$-predictable. Moreover, for $t \in[0, T]$, we use $\pi^{l}(t):=\frac{\phi^{l}(t) B_{t}}{V_{t-}^{\phi}}$ to denote the proportion of wealth invested in the lending account. We use $\pi^{b}(t):=\frac{\phi^{b}(t) \bar{B}_{t}}{V_{t-}^{\phi}}$ to denote the proportion of wealth invested in the borrowing account. Then $\pi^{l}(t) \geq 0, \pi^{b}(t) \leq 0$ and $\pi^{l}(t) \pi^{b}(t)=0$.

Recall (4). Using the self-financing condition, we can describe the wealth process as $V_{0}^{\phi}=v$ and

$$
d V_{t}^{\phi}=\phi(t) d\left(C_{t}+D_{t}\right)+V_{t}^{\phi} \pi^{b}(t) \frac{d \bar{B}_{t}}{\bar{B}_{t}}+V_{t}^{\phi} \pi^{l}(t) \frac{d B_{t}}{B_{t}} .
$$

We introduce $\psi(t)=\frac{\phi(t)}{V_{t-}^{\phi}}$ for the CDS security, obtained dividing the number of CDS shares by the current wealth level. Using (20), $\psi(t) C_{t}+\pi^{b}(t)+\pi^{l}(t)=1$ on $\tau_{I} \wedge T>t$. Using the conditions $\pi_{0}^{b}(t) \leq 0, \pi_{0}^{l}(t) \geq 0$ and $\pi_{0}^{b}(t) \pi_{0}^{l}(t)=0$, it follows that

$$
\pi^{b}(t)=\left\{1-\psi(t) C_{t}\right\}^{-}, \quad \pi^{l}(t)=\left\{1-\psi(t) C_{t}\right\}^{+}
$$

We used the notation $x^{-}:=\min \{x, 0\}$ and $x^{+}:=\max \{x, 0\}$ for $x \in \mathbb{R}$. We next define the class of admissible strategies for the investor.

Definition 1 Let $(t, v, \mathbf{z}) \in[0, T] \times \mathbb{R}_{+} \times \mathcal{S}$. The admissible control set $\tilde{\mathcal{U}}_{t}=$ $\tilde{\mathcal{U}}_{t}(v, \mathbf{z})$, is a class of $\mathbb{G}$-predictable locally bounded feedback trading strategies given by $\psi(u)=\psi_{\mathbf{H}(u-)}\left(u, V_{u-}^{v, \psi}\right)$ for $u \in[t, T]$. Here $V_{u}^{v, \psi}>0$ for $u \in[t, T]$ denotes the positive wealth process associated with the strategy $\psi$ when $V_{t}^{v, \psi}=v$ and $\mathbf{H}(t)=\mathbf{z}$. In particular, in the state $\mathbf{z}=\left(z_{1}, 1\right)$ with $z_{1} \in\{0,1\}$, we set the corresponding optimal feedback strategy to $\psi_{z_{1} 1}^{*}(u, v)=\pi_{z_{1} 1}^{l, *}(u, v)=\pi_{z_{1} 1}^{b, *}(u, v)=0$ for $(u, v) \in$ $[t, T] \times \mathbb{R}_{+}$. Here $\pi_{\mathbf{z}}^{l, *}(\cdot)$ and $\pi_{\mathbf{z}}^{b, *}(\cdot)$ denote the optimal feedback fractions of wealth invested in the borrowing and the lending account respectively. We use $\mathcal{U}_{t}$ to denote the set of all locally bounded feedback functions $\psi_{\mathbf{z}}(u, v)$ for $(u, v, z) \in[t, T] \times$ $\mathbb{R}_{+} \times \mathcal{S}$.

Using (13), it follows that 
Lemma 4 Let $\psi \in \tilde{\mathcal{U}}_{t}$ for $t \in[0, T]$. Then, the dynamics of the wealth $V_{u}^{\psi}$ for $u \in[t, T]$, is given by $V_{t}^{\psi}=v$ and

$$
\begin{aligned}
d V_{u}^{\psi}= & r V_{u}^{\psi} d u+V_{u}^{\psi}\left\{\psi(u)\left(\Gamma_{\mathbf{H}(u)}(u)-L\right) h_{1, \mathbf{H}(u)}(u)+\left(1-\psi(u) \Gamma_{\mathbf{H}(u)}(u)\right)\right. \\
& \left.-{ }^{-}\left(r_{\mathbf{H}(u)}(u)-r\right) \psi(u)\left[\Gamma_{\mathbf{H}^{I}(u)}(u)-\Gamma_{\mathbf{H}(u)}(u)\right]\left(1-H_{I}(u)\right) h_{I, \mathbf{H}(u)}(u)\right\} d u \\
& +V_{u-}^{\psi} \psi(u)\left(L-\Gamma_{\mathbf{H}(u-)}(u)\right) d H_{1}(u)+V_{u-}^{\psi} \psi(u)\left[\Gamma_{\mathbf{H}^{I}(u-)}(u)-\Gamma_{\mathbf{H}(u-)}(u)\right] d H_{I}(u) .
\end{aligned}
$$

\section{The optimal control problem}

Consider the power utility $U(v)=\gamma^{-1} v^{\gamma}$ for the initial wealth $v>0$ and the riskaversion parameter $\gamma \in(0,1)$. The value function of our optimal control problem is given by

$$
w_{\mathbf{z}}(t, v):=\sup _{\psi \in \tilde{\mathcal{U}}_{t}} \mathbb{E}^{\mathbb{P}}\left[U\left(V_{T \wedge \tau_{I}^{t}}^{\psi}\right) \mid V_{t}^{\psi}=v, \mathbf{H}(t)=\mathbf{z}\right] .
$$

Here the stopping time $\tau_{I}^{t}:=\inf \left\{s \geq t ; H_{I}(s)=1\right\}$.

Consider the default state $\mathbf{z}=\left(z_{1}, 1\right)$ for $z_{1} \in\{0,1\}$, i.e., corresponding to a defaulted investor. This implies that she will not invest in any security. Hence, her terminal wealth will be the same as her current wealth. As a result, the value function in this state is given by, for $z \in\{0,1\}$,

$$
w_{z_{1} 1}(t, v)=U(v), \forall(t, v) \in[0, T] \times \mathbb{R}_{+} .
$$

For the default state $\mathbf{z}=\left(z_{1}, 0\right)$ for $z_{1} \in\{0,1\}$, i.e. corresponding to the alive investor, using the above dynamic programming principle we obtain the following HJB equation in these states

$$
\sup _{\psi \in \mathcal{U}_{t}}\left(\frac{\partial}{\partial t}+\mathcal{L}_{v}^{\psi}+\mathcal{L}_{J}^{\psi}\right) w_{z_{1} 0}(t, v)=0
$$

with terminal condition $w_{z_{1} 0}(T, v)=U(v)$ for all $\left(v, z_{1}\right) \in \mathbb{R}_{+} \times\{0,1\}$. Here for $\varphi_{\mathbf{z}}(t, v)$ belonging to $C^{1}$ w.r.t. $(t, v)$, the operators

$$
\begin{aligned}
\mathcal{L}_{v}^{\psi} \varphi_{\mathbf{z}}(t, v):= & v \frac{\partial \varphi_{\mathbf{z}}(t, v)}{\partial v}\left\{r+\left(1-\psi \Gamma_{\mathbf{z}}(t)\right)^{-}\left(r_{\mathbf{z}}(t)-r\right)\right. \\
& \left.+\psi\left[\left(\Gamma_{\mathbf{z}}(t)-L\right) h_{1, \mathbf{z}}(t)-\left(\Gamma_{\mathbf{z}^{I}}(t)-\Gamma_{\mathbf{z}}(t)\right)\left(1-z_{I}\right) h_{I, \mathbf{z}}(t)\right]\right\},(27){ }^{\mathbb{P}}, \varphi_{1, \mathbf{z}}(t) \\
\mathcal{L}_{J}^{\psi} \varphi_{\mathbf{z}}(t, v):= & {\left[\varphi_{\mathbf{z}^{1}}\left(t, v+v \psi\left(L-\Gamma_{\mathbf{z}}(t)\right)\right)-\varphi_{\mathbf{z}}(t, v)\right]\left(1-z_{1}\right) h^{\mathbb{P}} } \\
& +\left[\varphi_{\mathbf{z}^{I}}\left(t, v+v \psi\left(\Gamma_{\mathbf{z}^{I}}(t)-\Gamma_{\mathbf{z}}(t)\right)\right)-\varphi_{\mathbf{z}}(t, v)\right]\left(1-z_{I}\right) h_{I, \mathbf{z}}^{\mathbb{P}}(t) .
\end{aligned}
$$

\section{Optimal feedback strategy}

The aim of this section is to find the optimal feedback strategy to our portfolio optimization problem (25)-(26). 
Let $\mathbf{z}=\left(z_{1}, z_{I}\right) \in \mathcal{S}$ be an arbitrary default state. Recall that at the state $z_{I}=1$ in which the credit event of the investor has occurred. Using Definition 1, the optimal feedback strategy $\psi_{z_{1} 1}^{*}(t)=\pi_{z_{1} 1}^{l, *}(t)=\pi_{z_{1} 1}^{b, *}(t)=0$. If $z_{1}=1$ and $z_{I}=0$, i.e., the CDS defaults but the investor's credit event has not yet occurred, then he can only invest in the risk-free asset and hence

$$
\psi_{10}^{*}(t)=0, \quad \pi_{10}^{b, *}(t)=0, \quad \pi_{10}^{l, *}(t)=1 .
$$

The nontrivial case is when no credit event has occurred, i.e. $\mathbf{z}=(0,0)$. We next characterize the optimal feedback strategy in this state. First, we postulate (and later verify) that the value function admitting $w_{\mathbf{z}}(t, v)=v^{\gamma} \varphi_{\mathbf{z}}(t)$ where $\varphi_{\mathbf{z}}(t)$ is a positive function which depends on state $\mathbf{z} \in \mathcal{S}$. It then follows directly from (25) that

$$
\varphi_{01}(t)=\varphi_{11}(t)=\gamma^{-1}, \quad t \in[0, T] .
$$

For $(\psi ; t, \varphi) \in \mathbb{R} \times[0, T] \times \mathbb{R}_{+}$, define

$$
\begin{aligned}
H(\psi ; t, \varphi):= & \gamma\left\{r+\left(1-\psi \Gamma_{00}(t)\right)^{-}\left(r_{00}(t)-r\right)\right. \\
& \left.+\psi\left[\left(\Gamma_{00}(t)-L\right) h_{1,00}(t)-\left(\Gamma_{01}(t)-\Gamma_{00}(t)\right) h_{I, 00}(t)\right]\right\} \varphi \\
& +\left\{\left[1+\psi\left(L-\Gamma_{00}(t)\right)\right]^{\gamma} \varphi_{10}(t)-\varphi\right\} h_{1,00}^{\mathbb{P}}(t) \\
& +\left\{\left[1+\psi\left(\Gamma_{01}(t)-\Gamma_{00}(t)\right)\right]^{\gamma} \varphi_{01}(t)-\varphi\right\} h_{I, 00}^{\mathbb{P}}(t) .
\end{aligned}
$$

Equation (26) in the state $\mathbf{z}=(0,0)$ can then be rewritten as

$$
\varphi^{\prime}(t)+\sup _{\psi \in \mathbb{R}} H(\psi ; t, \varphi(t))=0 .
$$

The aim is to analyze $\psi \rightarrow H(\psi ; t, \varphi)$ for characterizing the optimal feedback strategy $\psi^{*}$ in the state $\mathbf{z}=(0,0)$. Noting that for fixed $(t, \varphi) \in[0, T] \times \mathbb{R}_{+}$, $H(\psi ; t, \varphi)$ is not differentiable at $\psi=\ell(t):=\Gamma_{00}(t)^{-1}>0$. Hence the first-order condition for optimality cannot be applied. To this purpose, we introduce

$$
\begin{aligned}
g_{1}(\psi ; t, \varphi):= & {\left[\left(\Gamma_{00}(t)-L\right) h_{1,00}(t)-\left(\Gamma_{01}(t)-\Gamma_{00}(t)\right) h_{I, 00}(t)\right] \varphi } \\
& +\left[1+\psi\left(L-\Gamma_{00}(t)\right)\right]^{\gamma-1}\left(L-\Gamma_{00}(t)\right) h_{1,00}^{\mathbb{P}}(t) \varphi_{10}(t) \\
& +\left[1+\psi\left(\Gamma_{01}(t)-\Gamma_{00}(t)\right)\right]^{\gamma-1}\left(\Gamma_{01}(t)-\Gamma_{00}(t)\right) h_{I, 00}^{\mathbb{P}}(t) \varphi_{01}(t), \\
g_{2}(\psi ; t, \varphi):= & g_{1}(\psi ; t, \varphi)-\Gamma_{00}(t)\left(r_{00}(t)-r\right) \varphi .
\end{aligned}
$$

It can be seen from (32) that both $g_{1}$ and $g_{2}$ are strictly decreasing and continuous in $\psi$. On the other hand, to guarantee that the wealth process is always positive after a default in terms of Eq. (23), the feedback strategy $\psi$ should satisfy the following admissibility condition:

$$
\psi=\psi(t, \varphi)>\max \left\{-\frac{1}{L-\Gamma_{00}(t)},-\frac{1}{\Gamma_{01}(t)-\Gamma_{00}(t)}\right\}=-\frac{1}{L-\Gamma_{00}(t)},
$$

where the last equality above follows from Lemma 1.

We next characterize the optimal feedback strategy satisfying (33). The key idea behind our method is to decompose the function as

$$
H(\psi ; t, \varphi)=\mathbf{1}_{\psi \leq \ell(t)} H_{1}(\psi ; t, \varphi)+\mathbf{1}_{\psi>\ell(t)} H_{2}(\psi ; t, \varphi) .
$$


Here the decomposing functions

$$
\begin{aligned}
H_{1}(\psi ; t, \varphi):= & \gamma\left\{r+\psi\left[\left(\Gamma_{00}(t)-L\right) h_{1,00}(t)-\left(\Gamma_{01}(t)-\Gamma_{00}(t)\right) h_{I, 00}(t)\right]\right\} \varphi \\
& +\left\{\left[1+\psi\left(L-\Gamma_{00}(t)\right)\right]^{\gamma} \varphi_{10}(t)-\varphi\right\} h_{1,00}^{\mathbb{P}}(t) \\
& +\left\{\left[1+\psi\left(\Gamma_{01}(t)-\Gamma_{00}(t)\right)\right]^{\gamma} \varphi_{01}(t)-\varphi\right\} h_{I, 00}^{\mathbb{P}}(t), \\
H_{2}(\psi ; t, \varphi):= & H_{1}(\psi ; t, \varphi)+\gamma\left(1-\psi \Gamma_{00}(t)\right)\left(r_{00}(t)-r\right) \varphi .
\end{aligned}
$$

It is easy to see that $g_{i}$ is the first-order derivative of $\gamma^{-1} H_{i}$ w.r.t. $\psi$ for $i=1,2$. Then we have the following lemma whose proof is reported in the Appendix.

Lemma 5 Let $(t, \varphi) \in[0, T] \times \mathbb{R}_{+}$be fixed. For each $i=1,2$, there exists a unique finite $\psi_{i}^{\text {foc }}>-\frac{1}{L-\Gamma_{00}(t)}$ satisfying (33) such that $g_{i}\left(\psi_{i}^{\text {foc }} ; t, \varphi\right)=0$. Moreover, $\psi_{i}^{f o c}$, viewed as a function of $(t, \varphi)$, is $C^{1}$. In addition, for all $(t, \varphi) \in$ $[0, T] \times \mathbb{R}_{+}$it holds that

$$
-\frac{1}{L-\Gamma_{00}(t)}<\psi_{2}^{f o c}(t, \varphi)<\psi_{1}^{f o c}(t, \varphi)<+\infty .
$$

Using Lemma 5, we characterize the optimal feedback strategy in terms of a relation between the critical point $\ell(t)$ and the solutions $\psi_{i}^{f o c}$ of the system of first order condition equations.

Proposition 1 For $i=1,2$ and $(t, \varphi) \in[0, T] \times \mathbb{R}_{+}$, let $\psi_{i}^{f o c}=\psi_{i}^{f o c}(t, \varphi)$ be as obtained in Lemma 5 above. Then in the state $\mathbf{z}=(0,0)$,

(i) The optimum $\psi^{*}(t, \varphi)=\psi_{2}^{f o c}(t, \varphi)$ if and only if the critical point $\ell(t) \leq$ $\psi_{2}^{f o c}(t, \varphi)$. Correspondingly, the optimal feedback borrowing strategy is given by $\pi^{b, *}(t, \varphi)=1-\psi_{2}^{f o c}(t, \varphi) \Gamma_{00}(t)$ and the optimal feedback lending strategy is $\pi^{l, *}(t, \varphi)=0$;

(ii) The optimum $\psi^{*}(t, \varphi)=\ell(t)$ if and only if the critical point $\psi_{2}^{\text {foc }}(t, \varphi) \leq$ $\ell(t) \leq \psi_{1}^{f o c}(t, \varphi)$. Correspondingly, the optimal feedback borrowing and lending strategies are given by $\pi^{b, *}(t, \varphi)=\pi^{l, *}(t, \varphi)=0$;

(iii) The optimum $\psi^{*}(t, \varphi)=\psi_{1}^{\text {foc }}(t, \varphi)$ if and only if the critical point $\ell(t) \geq$ $\psi_{1}^{f o c}(t, \varphi)$. Correspondingly, the optimal feedback borrowing strategy is given by $\pi^{b, *}(t, \varphi)=0$ and the optimal feedback lending strategy is $\pi^{l, *}(t, \varphi)=$ $1-\psi_{1}^{f o c}(t, \varphi) \Gamma_{00}(t)$.

Proof For fixed $(t, \varphi) \in[0, T] \times \mathbb{R}_{+}$, recall the decomposition (34) with $H_{i}$, $i=1,2$ defined in (32). We first prove the necessity. Consider the case (i). In this case, $g_{1}(\psi ; t, \varphi)>0$ for all $\psi \leq \ell(t)$. This implies that the continuous function $H_{1}(\psi ; t, \varphi)$ is increasing w.r.t. $\psi \leq \ell(t)$. Moreover, for $\psi>\ell(t), \psi_{2}^{f o c}(t, \varphi)$ is the unique local optimum for the continuous function $\psi \rightarrow \mathrm{H}_{2}(\psi ; t, \varphi)$ using Lemma 5. Since in case (i) $\psi_{2}^{f o c}(t, \varphi)>\ell(t)$, we also have that the continuous function $\psi \rightarrow H_{2}(\psi ; t, \varphi)$ is increasing for $\ell(t) \leq \psi \leq \psi_{2}^{f o c}(t, \varphi)$, and decreasing 
when $\psi>\psi_{2}^{f o c}(t, \varphi)$. This also implies that $\psi^{*}(t, \varphi)=\psi_{2}^{f o c}(t, \varphi)$ (see Fig. 1). On the other hand, since $\psi_{2}^{f o c}(t, \varphi)>\ell(t)$ in case (i), then the optimal fraction invested in the borrowing account is given by $\pi^{b, *}(t, \varphi)=\left(1-\psi^{*}(t, \varphi) \Gamma_{00}(t)\right)^{-}=$ $\left(1-\psi_{2}^{f o c}(t, \varphi) \Gamma_{00}(t)\right)^{-}=1-\psi_{2}^{f o c}(t, \varphi) \Gamma_{00}(t)<0$. Hence, the optimal fraction of wealth in the lending account is $\pi^{l, *}(t, \varphi)=0$ using (22).

For case (ii), $g_{1}(\psi ; t, \varphi)>0$ for all $\psi \leq \ell(t)$, and $g_{2}(\psi ; t, \varphi)<0$ for all $\psi \geq$ $\ell(t)$. This implies that the continuous function $H_{1}(\psi ; t, \varphi)$ is increasing w.r.t. $\psi \leq$ $\ell(t)$, while the continuous function $H_{2}(\psi ; t, \varphi)$ is decreasing w.r.t. $\psi \geq \ell(t)$. Since $\psi_{2}^{f o c}(t, \varphi)<\ell(t)<\psi_{1}^{f o c}(t, \varphi)$ in case (ii), then $\psi^{*}(t, \varphi)=\ell(t)$ (see Fig. 2). On the other hand, in terms of (22), the optimal fraction invested in the borrowing account is given by $\pi^{b, *}(t, \varphi)=\left(1-\psi^{*}(t, \varphi) \Gamma_{00}(t)\right)^{-}=\left(1-\ell(t) \Gamma_{00}(t)\right)^{-}=$ 0 , and the optimal fraction invested in the lending account is $\pi^{l, *}(t, \varphi)=(1-$ $\left.\psi^{*}(t, \varphi) \Gamma_{00}(t)\right)^{+}=0$.

For case (iii), $g_{2}(\psi ; t, \varphi)<0$ for all $\psi \geq \ell(t)$. This implies that the continuous function $\mathrm{H}_{2}(\psi ; t, \varphi)$ is decreasing w.r.t. $\psi \geq \ell(t)$. Note that for $\psi<\ell(t)$, using Lemma 5, the continuous function $\psi \rightarrow H_{1}(\psi ; t, \varphi)$ admits a unique local optimum $\psi_{1}^{f o c}(t, \varphi)$. Since in case (iii), $\ell(t)>\psi_{1}^{f o c}(t, \varphi)$, the continuous function $\psi \rightarrow H_{1}(\psi ; t, \varphi)$ is decreasing when $\psi_{1}^{f o c}(t, \varphi) \leq \psi \leq \ell(t)$, and increasing when $\psi<\psi_{1}^{f o c}(t, \varphi)$. Consequently, the optimum of $\psi \rightarrow H(\psi ; t, \varphi)$ is $\psi_{1}^{f o c}(t, \varphi)$ (see Fig. 3). On the other hand, since $\ell(t)>\psi_{1}^{f o c}(t, \varphi)$, the optimal fraction of wealth invested in the borrowing account is given by $\pi^{b, *}(t, \varphi)=$ $\left(1-\psi_{1}^{f o c}(t, \varphi) \Gamma_{00}(t)\right)^{-}=0$, and hence the optimal fraction of wealth invested in the lending account is $\pi^{l, *}(t, \varphi)=\left(1-\psi_{1}^{f o c}(t, \varphi) \Gamma_{00}(t)\right)^{+}=1-\psi_{1}^{f o c}(t, \varphi) \Gamma_{00}(t)$ using (22).

We next check sufficiency. For case (i), given that the optimum $\psi^{*}(t, \varphi)=$ $\psi_{2}^{f o c}(t, \varphi)$, assume by contradiction that the critical point $\ell(t)>\psi_{2}^{f o c}(t, \varphi)$. Consider first the case where $\psi_{2}^{f o c}(t, \varphi)<\ell(t)<\psi_{1}^{f o c}(t, \varphi)$. Then, using the
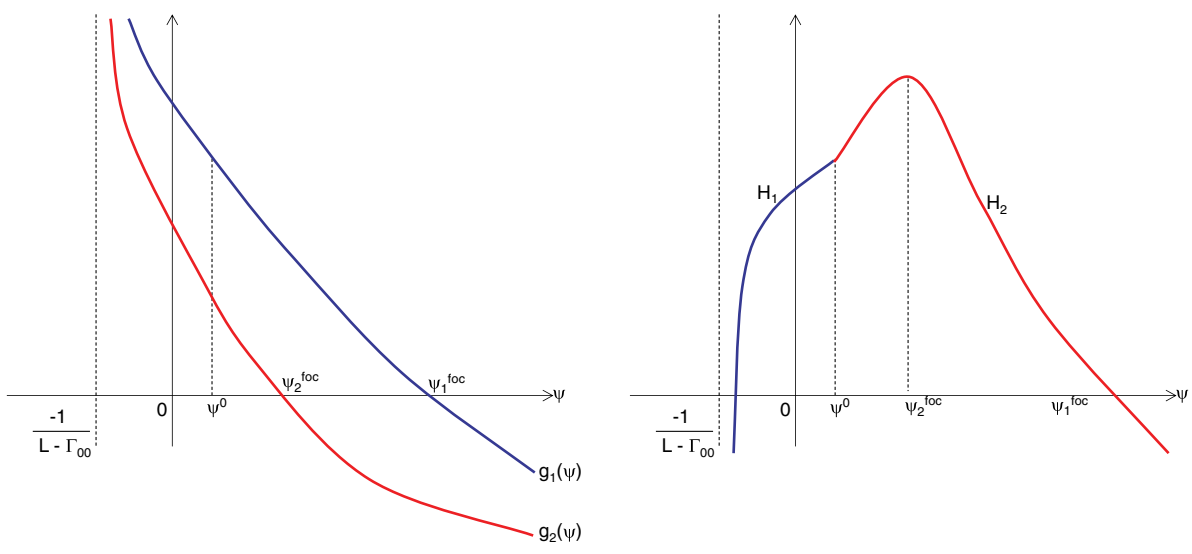

Fig. 1 The optimum $\psi^{*}(t, \varphi)$ in case (i) 

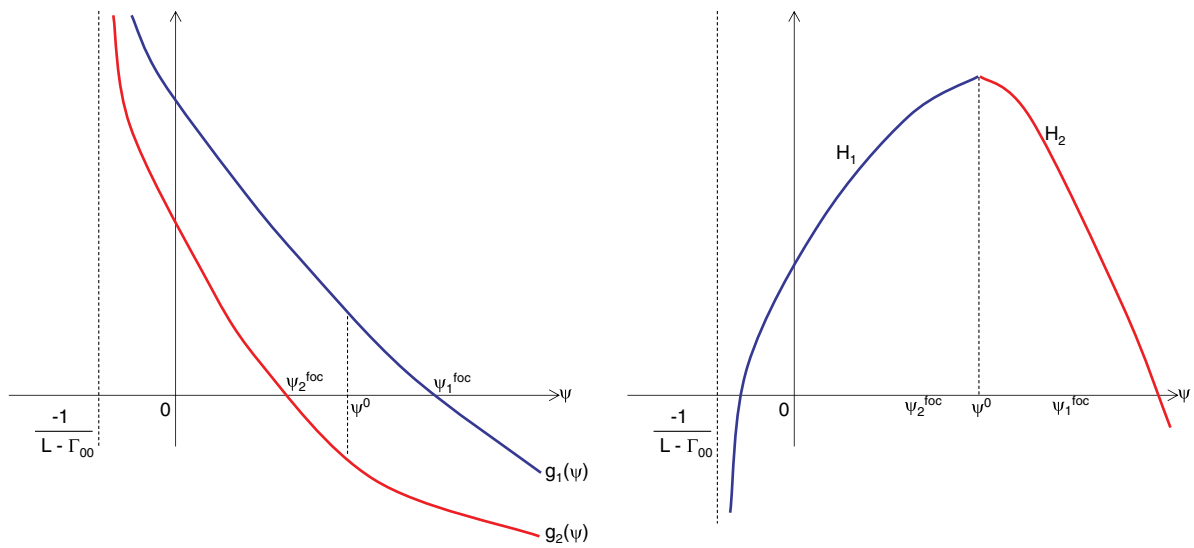

Fig. 2 The optimum $\psi^{*}(t, \varphi)$ in case (ii)

necessary result proved above in (ii), it follows that the optimum $\psi^{*}(t, \varphi)=$ $\ell(t)$, which contradicts the given assumption $\psi^{*}(t, \varphi)=\psi_{2}^{f o c}(t, \varphi)$, since $\ell(t)>\psi_{2}^{f o c}(t, \varphi)$ in this subcase. Next consider the remaining case where $\ell(t) \geq \psi_{1}^{f o c}(t, \varphi)$. Using the necessary result proved above in (iii), we conclude that the optimum $\psi^{*}(t, \varphi)=\psi_{1}^{f o c}(t, \varphi)$, which also contradicts the given assumption $\psi^{*}(t, \varphi)=\psi_{2}^{f o c}(t, \varphi)$ due to the inequality (36) in Lemma 5. Thus we have proven sufficiency in case (i). For cases (ii) and (iii), the corresponding proofs are similar. Here we omit them. This completes the proof of the proposition.

Lemma 5 provides a finite lower bound for $\psi_{i}^{f o c}$. The next lemma gives a finite upper bound for $\psi_{i}^{f o c}$. These boundedness estimates will play a crucial role in proving existence and uniqueness of solutions of the HJB equation.
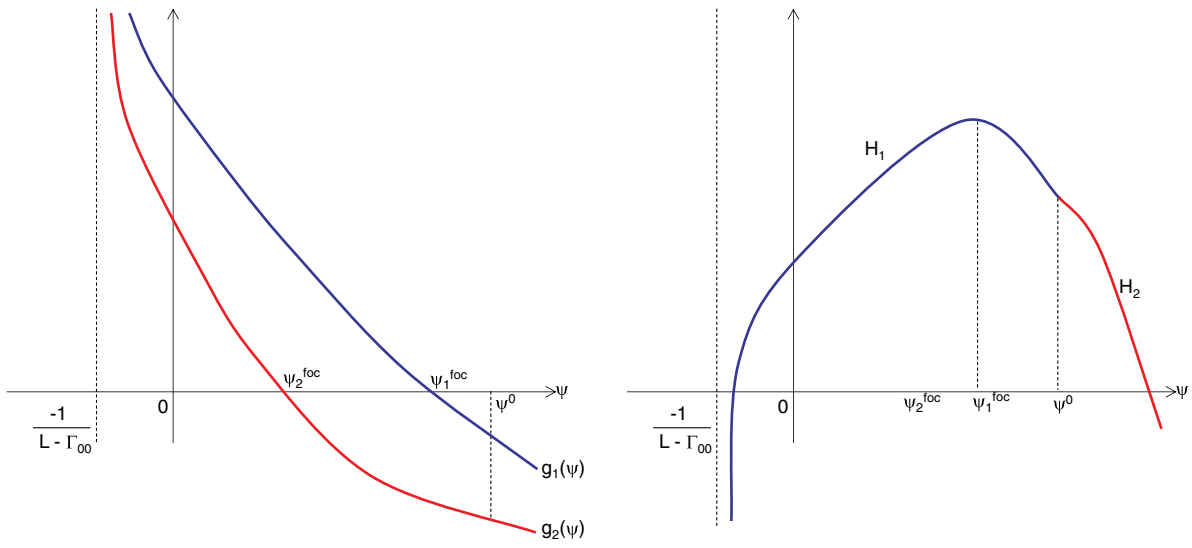

Fig. 3 The optimum $\psi^{*}(t, \varphi)$ in case (iii) 
Lemma 6 For $(t, \varphi) \in[0, T] \times \mathbb{R}_{+}$, it holds that

$$
-\frac{1}{L-\Gamma_{00}(t)}<\psi_{2}^{f o c}(t, \varphi)<\psi_{1}^{f o c}(t, \varphi) \leq \max \{\bar{\psi}(t, \varphi), \hat{\psi}(t, \varphi)\},
$$

where

$$
\begin{aligned}
& \bar{\psi}(t, \varphi):=\frac{1}{L-\Gamma_{00}(t)}\left[\left(\frac{h_{1,00}(t) \varphi}{h_{1,00}^{\mathbb{P}}(t) \varphi_{10}(t)}\right)^{\frac{1}{\gamma-1}}-1\right], \\
& \hat{\psi}(t, \varphi):=\frac{1}{\Gamma_{01}(t)-\Gamma_{00}(t)}\left[\left(\frac{h_{I, 00}(t) \varphi}{h_{I, 00}^{\mathbb{P}}(t) \varphi_{01}(t)}\right)^{\frac{1}{\gamma-1}}-1\right] .
\end{aligned}
$$

The proof is delegated into the Appendix.

\section{Solvability of HJB equations}

We prove the existence and uniqueness of a global solution to HJB equations at each default state. We then show that it corresponds with the value function of the control problem via a verification theorem.

Recall (29) and consider $z_{1}=1$ in Eq. (26) with the optimum given by (28). We obtain the HJB equation at default state $\mathbf{z}=(1,0)$ which given by

$$
0=\varphi_{10}^{\prime}(t)+\gamma r \varphi_{10}(t)+\left(\varphi_{11}(t)-\varphi_{10}(t)\right) h_{I, 10}^{\mathbb{P}}(t)
$$

with terminal condition $\varphi_{10}(T)=\gamma^{-1}$. The closed-form solution is given by, for $t \in[0, T]$,

$$
\varphi_{10}(t)=\gamma^{-1}\left(1+\int_{t}^{T} h_{I, 10}^{\mathbb{P}}(s) e^{\int_{s}^{T}\left(h_{I, 10}^{\mathbb{P}}(u)-\gamma r\right) d u} d s\right) e^{-\int_{t}^{T}\left(h_{I, 10}^{\mathbb{P}}(u)-\gamma r\right) d u} .
$$

We next analyze the nontrivial case, i.e. when no credit event has occurred. To this purpose, recall $\psi_{i}^{f o c}=\psi_{i}^{f o c}(t, \varphi)$ as obtained in Lemma 5. We have

Lemma 7 For each $i=1,2$, as a $C^{1}$ function, $\varphi \rightarrow \psi_{i}^{f o c}(t, \varphi)$ is strictly decreasing for each fixed time $t \in[0, T]$. Moreover, for each $t \in[0, T]$,

$$
\lim _{\varphi \downarrow 0} \psi_{i}^{f o c}(t, \varphi)=+\infty, \quad \lim _{\varphi \uparrow \infty} \psi_{i}^{f o c}(t, \varphi)=-\frac{1}{L-\Gamma_{00}(t)} .
$$

Recall that $\ell(t)=\Gamma_{00}(t)^{-1}>0$ for all $t \in[0, T]$. Then, using Lemma 7 above, it follows that for each fixed time $t \in[0, T]$, there exists a unique $b_{i}(t)>0$ such that $\ell(t)=\psi_{i}^{f o c}\left(t, b_{i}(t)\right)$ for $i=1,2$. Moreover, $t \rightarrow b_{i}(t)$ is continuous and $b_{2}(t)<b_{1}(t)$ for all $t \in[0, T]$ using Lemma 6. From Proposition 1, it follows that the optimum $\psi^{*}=\psi^{*}(t, \varphi)$ is a continuous function of $(t, \varphi) \in[0, T] \times \mathbb{R}_{+}$, 
and admits the following representation (see also Fig. 4 for an illustration). For each $t \in[0, T]$ fixed, on $\varphi \in \mathbb{R}_{+}$,

$$
\psi^{*}(t, \varphi)=\left\{\begin{array}{cc}
\psi_{2}^{f o c}(t, \varphi), & \varphi \leq b_{2}(t), \\
\ell(t), & \varphi \in\left(b_{2}(t), b_{1}(t)\right), \\
\psi_{1}^{f o c}(t, \varphi), & \varphi \geq b_{1}(t) .
\end{array}\right.
$$

It implies that for fixed $t \in[0, T], \varphi \rightarrow \psi^{*}(t, \varphi)$ is continuous and decreasing. It is not difficult to claim that

Lemma 8 It holds that $\varphi \rightarrow \psi^{*}(t, \varphi)$ given by (41) is locally Lipschitz-continuous uniformly in $t \in[0, T]$.

We next focus on the solvability of Eq. (31). To this purpose, we rewrite it as in the following form

$$
\varphi^{\prime}(t)+A\left(t, \psi^{*}(t, \varphi(t))\right) \varphi(t)+C\left(t, \psi^{*}(t, \varphi(t))\right)=0
$$

with terminal condition $\varphi(T)=\gamma^{-1}$, where $\psi^{*}(t, \varphi)$ is given by (41), and the coefficients

$$
\begin{aligned}
A(t, \psi):= & \gamma\left\{r+\left(1-\psi \Gamma_{00}(t)\right)^{-}\left(r_{00}(t)-r\right)\right. \\
& \left.-\psi\left[\left(L-\Gamma_{00}(t)\right) h_{1,00}(t)+\left(\Gamma_{01}(t)-\Gamma_{00}(t)\right) h_{I, 00}(t)\right]\right\} \\
& -h_{1,00}^{\mathbb{P}}(t)-h_{I, 00}^{\mathbb{P}}(t), \\
C(t, \psi):= & {\left[1+\psi\left(L-\Gamma_{00}(t)\right)\right]^{\gamma} h_{1,00}^{\mathbb{P}}(t) \varphi_{10}(t) } \\
& +\gamma^{-1}\left[1+\psi\left(\Gamma_{01}(t)-\Gamma_{00}(t)\right)\right]^{\gamma} h_{I, 00}^{\mathbb{P}}(t) .
\end{aligned}
$$

Above, we have used the explicit representation for $\varphi_{01}(t)=\gamma^{-1}$ given in (29), and that $\varphi_{10}(t)$ is given by (39).

Fig. 4 The dependence of $\psi^{*}(t, \varphi)$ on $\varphi$

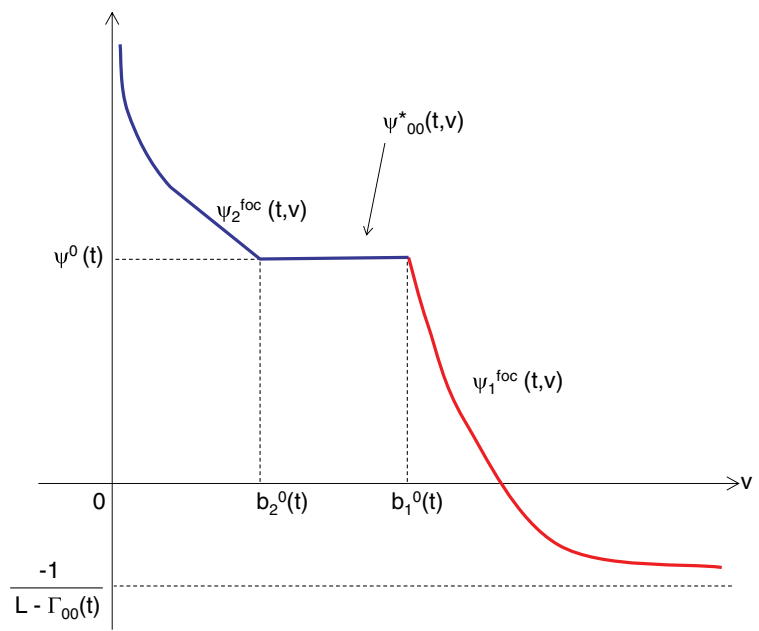


Noting that $\psi^{*}(t, \varphi)$ is the optimum. Then, for all $(t, \varphi) \in[0, T] \times \mathbb{R}_{+}$,

$$
A\left(t, \psi^{*}(t, \varphi)\right) \varphi+C\left(t, \psi^{*}(t, \varphi)\right) \geq A(t, \ell(t)) \varphi+C(t, \ell(t)) .
$$

Consider the following ODE

$$
u^{\prime}(t)+A(t, \ell(t)) u(t)+C(t, \ell(t))=0
$$

with terminal condition $u(T)=a_{0} \in\left(0, \gamma^{-1}\right]$. We rewrite Eq. (42) as

$$
v^{\prime}(t)+\bar{A}(t, v(t)) v(t)+\bar{C}(t, v(t))=0
$$

with $v(T)=\gamma^{-1}$, where $\bar{A}(t, v):=A\left(t, \psi^{*}(t, v)\right)$ and $\bar{C}(t, v):=C\left(t, \psi^{*}(t, v)\right)$ on $(t, v) \in[0, T] \times \mathbb{R}_{+}$. The coefficients $\bar{A}(t, v)$ and $\bar{C}(t, v)$ admit the following estimates.

Lemma 9 Let $(t, v) \in[0, T] \times \mathbb{R}_{+}$. Then there exist $K, \bar{K}>0$ such that $|\bar{A}(t, v)| \leq K+\bar{K} v^{\frac{1}{\gamma-1}}$ and $0<\bar{C}(t, v) \leq K+\bar{K} v^{\frac{\gamma}{\gamma-1}}$.

The following lemma, proven in the Appendix, show that the solution to the HJB equation is bounded from below.

Lemma 10 If there exists a solution $(v(t) ; t \in[0, T])$ to Eq. (46), then there exists a constant $\eta>0$ such that $v(t) \geq \eta$ for all $t \in[0, T]$.

Using Lemma 10, and recall $\eta$ therein, Eq. (46) is equivalent to the following equation

$$
v^{\prime}(t)+\bar{A}_{\eta}(t, v(t)) v(t)+\bar{C}_{\eta}(t, v(t))=0
$$

with $v(T)=\gamma^{-1}$. Here, for $(t, v) \in[0, T] \times \mathbb{R}_{+}$, the coefficients

$$
\bar{A}_{\eta}(t, v):=\bar{A}(t, v \vee \eta), \quad \bar{C}_{\eta}(t, v):=\bar{C}(t, v \vee \eta) .
$$

We hence focus on the existence and uniqueness of the solution to Eq. (47). For this purpose, given $\eta$ in Lemma 10 and $K, \bar{K}$ in Lemma 9, define $f_{1}(\eta):=K+$ $\bar{K} \eta^{\frac{1}{\gamma-1}}, f_{2}(\eta):=K+\bar{K} \eta^{\frac{\gamma}{\gamma-1}}$, and the following positive deterministic function by

$$
\bar{\kappa}(t):=\left(\frac{1}{\gamma}+\frac{f_{2}(\eta)}{f_{1}(\eta)}\right) e^{f_{1}(\eta)(T-t)}-\frac{f_{2}(\eta)}{f_{1}(\eta)}, \quad t \in[0, T] .
$$

Then we have

Theorem 1 There exists a unique solution $(v(t) ; t \in[0, T])$ to Eq. (47). Moreover, $\eta \leq v(t) \leq \bar{\kappa}(t)$ for all $t \in[0, T]$.

Proof Consider the truncated coefficients given by, for $(t, v) \in[0, T] \times \mathbb{R}_{+}$,

$$
\bar{A}_{\eta, \kappa}(t, v):=\bar{A}_{\eta}(t, v \wedge \bar{\kappa}(t)), \quad \bar{C}_{\eta, \kappa}(t, v):=\bar{C}_{\eta}(t, v \wedge \bar{\kappa}(t)) .
$$

Using the estimates in Lemma 9, we have that for all $(t, v) \in[0, T] \times \mathbb{R}_{+}$,

$$
\left|\bar{A}_{\eta, \kappa}(t, v)\right| \leq f_{1}(\eta), \quad 0<\bar{C}_{\eta, \kappa}(t, v) \leq f_{2}(\eta) .
$$


Recall $A(t, \psi)$ and $C(t, \psi)$ defined in (43). Then, $\psi \rightarrow A(t, \psi)$ is Lipschitzcontinuous and $\psi \rightarrow C(t, \psi)$ is locally Lipschitz-continuous uniformly in time $t \in[0, T]$. Then using Lemma 8 and the estimate (51), the truncated coefficients $v \rightarrow \bar{A}_{\eta, \kappa}(t, v)$ and $v \rightarrow \bar{C}_{\eta, \kappa}(t, v)$ are (bounded) Lipschitz-continuous on $v \in \mathbb{R}_{+}$, uniformly in time $t \in[0, T]$. This yields that the following truncated ODE

$$
v_{\kappa}^{\prime}(t)+\bar{A}_{\eta, \kappa}\left(t, v_{\kappa}(t)\right) v_{\kappa}(t)+\bar{C}_{\eta, \kappa}\left(t, v_{\kappa}(t)\right)=0, \quad v_{\kappa}(T)=\gamma^{-1} .
$$

admits a unique solution $v_{\kappa}(t)$ for $t \in[0, T]$. On the other hand, we can write Eq. (52) in the following integral form, i.e., for each $t \in[0, T]$,

$$
v_{\kappa}(t)=\gamma^{-1} e^{\int_{t}^{T} \bar{A}_{\eta, \kappa}\left(s, v_{\kappa}(s)\right) d s}+\int_{t}^{T} \bar{C}_{\eta, \kappa}\left(s, v_{\kappa}(s)\right) e^{\int_{t}^{s} \bar{A}_{\eta, \kappa}\left(\iota, v_{\kappa}(\iota)\right) d \iota} d s .
$$

Using (51), it follows that for all $t \in[0, T]$,

$$
\begin{aligned}
v_{\kappa}(t) & =\gamma^{-1} e^{\int_{t}^{T} \bar{A}_{\eta, \kappa}\left(s, v_{\kappa}(s)\right) d s}+\int_{t}^{T} \bar{C}_{\eta, \kappa}\left(s, v_{\kappa}(s)\right) e^{\int_{t}^{s} \bar{A}_{\eta, \kappa}\left(\iota, v_{\kappa}(\iota)\right) d \iota} d s \\
& \leq \gamma^{-1} e^{l_{1}(\eta)(T-t)}+l_{2}(\eta) \int_{t}^{T} e^{l_{1}(\eta)(s-t)} d s=\bar{\kappa}(t) .
\end{aligned}
$$

In terms of (50), it holds that

$$
\bar{A}_{\eta, \kappa}\left(t, v_{\kappa}(t)\right)=\bar{A}_{\eta}\left(t, v_{\kappa}(t)\right), \quad \bar{C}_{\eta, \kappa}\left(t, v_{\kappa}(t)\right)=\bar{C}_{\eta}\left(t, v_{\kappa}(t)\right) .
$$

By the uniqueness of the solution to Eq. (52), we have $v(t)=v_{\kappa}(t)$ for all $t \in[0, T]$, and moreover $\eta \leq v(t) \leq \bar{\kappa}(t)$ for all $t \in[0, T]$ in light of (53) and Lemma 10. This completes the proof of the theorem.

We finally mention that the verification theorem also holds on our control problem, i.e., the solution of the HJB equation is the value function of our control problem. The proof is standard and it heavily depends on the bounded solutions to the HJB equations discussed above. Hence, we omit the statement of the verification theorem and the proof.

\section{Appendix}

\section{Technical proofs}

Proof of Lemma 3 We first consider the limit expression for $r_{10}(T)$. Using (18),

$$
\begin{aligned}
\lim _{t \uparrow T} \frac{1}{T-t} \log \left(\frac{1}{\Phi_{10}(t, T)}\right) & =\lim _{t \uparrow T} \frac{1}{T-t} \int_{t}^{T} h_{I, 10}(u) d u \\
& =\lim _{x \downarrow 0} \frac{1}{x} \int_{0}^{x} h_{I, 10}(T-u) d u=r+h_{I, 10}(T) .
\end{aligned}
$$

For the limit $r_{00}(T)$, we set $x=T-t$ and rewrite

$$
\Phi_{10}(t, T)=\tilde{\Phi}_{I, 10}(x):=e^{-\int_{0}^{x}\left(r+h_{I, 10}(T-u)\right) d u},
$$


and

$$
\begin{aligned}
\Phi_{00}(t, T)= & \tilde{\Phi}_{I, 00}(x):=e^{-\int_{0}^{x}\left(r+h_{1,00}(T-u)+h_{I, 00}(T-u)\right) d u} \\
& +\tilde{\Phi}_{I, 10}(x) \int_{0}^{x} h_{1,00}(T-u) e^{-\int_{u}^{x} h_{1,00}(T-s) d s} e^{\int_{u}^{x}\left(h_{I, 10}(T-s)-h_{I, 00}(T-s)\right) d s} d u .
\end{aligned}
$$

Moreover, the derivative function $\tilde{\Phi}_{I, 00}^{\prime}(x)$ is given by

$$
\begin{aligned}
\tilde{\Phi}_{I, 00}^{\prime}(x)= & -\left(r+h_{1,00}(T-x)+h_{I, 00}(T-x)\right) e^{-\int_{0}^{x}\left(r+h_{1,00}(T-u)+h_{I, 00}(T-u)\right) d u} \\
& +\tilde{\Phi}_{I, 10}^{\prime}(x) \int_{0}^{x} h_{1,00}(T-u) e^{-\int_{u}^{x} h_{1,00}(T-s) d s} e^{\int_{u}^{x}\left(h_{I, 10}(T-s)-h_{I, 00}(T-s)\right) d s} d u \\
& +\tilde{\Phi}_{I, 10}(x) h_{1,00}(T-x) .
\end{aligned}
$$

Note that $\tilde{\Phi}_{I, 00}(0)=\tilde{\Phi}_{I, 10}(0)=1$. Then

$$
\lim _{x \downarrow 0} \tilde{\Phi}_{I, 00}^{\prime}(x)=-\left(r+h_{1,00}(T)+h_{I, 00}(T)\right)+h_{1,00}(T)=-\left(r+h_{I, 00}(T)\right) .
$$

By application of L'Hospital's rule, it follows that

$$
\lim _{t \uparrow T} \frac{1}{T-t} \log \left(\frac{1}{\Phi_{00}(t, T)}\right)=\lim _{x \downarrow 0}-\frac{\log \left(\tilde{\Phi}_{I, 00}(x)\right)}{x}=-\lim _{x \downarrow 0} \frac{\tilde{\Phi}_{I, 00}^{\prime}(x)}{\tilde{\Phi}_{I, 00}(x)}=r+h_{I, 00}(T) .
$$

Using (18), the borrowing rate $r_{10}(t)$ satisfies, for $t \in[0, T)$,

$$
r_{10}(t)=\frac{1}{T-t} \int_{t}^{T}\left(r+h_{I, 10}(u)\right) d u,
$$

and $r_{10}(T)=r+h_{I, 10}(T)$ using Lemma 3. This yields the estimate

$$
r+\underline{m}_{I, 10} \leq r_{10}(t) \leq r+\bar{m}_{I, 10}, \quad t \in[0, T] .
$$

Noting that

$\Phi_{00}(t, T)=e^{-r(T-t)-\int_{t}^{T} h_{I, 00}(u) d u}+\int_{t}^{T_{1}}\left[h_{1,00}(s)\left(\Phi_{10}(s, T)-\Phi_{00}(s, T)\right)\right] e^{-\int_{t}^{s} r d u} d s$.

It then follows from (18) that

$$
\Phi_{00}(t, T)<e^{-r(T-t)-\int_{t}^{T} h_{I, 00}(u) d u} \leq e^{-\left(r+\underline{m}_{I, 00}\right)(T-t)}, \quad t \in[0, T) .
$$

On the other hand, it holds that

$\Phi_{00}(t, T)>e^{-\int_{t}^{T}\left(r+h_{1,00}(u)+h_{I, 00}(u)\right) d u} \geq e^{-\left(r+\bar{m}_{1,00}+\bar{m}_{I, 00}\right)(T-t)}, \quad t \in[0, T)$.

Using (54) and (55), for $t \in[0, T)$, we obtain

$$
\left(r+\underline{m}_{I, 00}\right)(T-t)<\log \left(\frac{1}{\Phi_{00}(t, T)}\right)<\left(r+\bar{m}_{1,00}+\bar{m}_{I, 00}\right)(T-t) .
$$

This yields that, for all $t \in[0, T)$,

$$
r+\underline{m}_{I, 00}<\frac{1}{T-t} \log \left(\frac{1}{\Phi_{00}(t, T)}\right)<r+\bar{m}_{1,00}+\bar{m}_{I, 00} .
$$

Note that $r_{00}(T)=r+h_{I, 00}(T)$ using Lemma 3 . Then, we obtain the estimate

$$
r+\underline{m}_{I, 00} \leq r_{00}(t)<r+\bar{m}_{1,00}+\bar{m}_{I, 00}, \quad t \in[0, T) .
$$


This concludes the proof of the lemma.

Proof of Lemma 5 Recall $g_{1}(\psi ; t, \varphi)$ defined by (32). For all $\psi$ satisfying (33), $g_{1}(\psi ; t, \varphi)$ is continuous and decreasing w.r.t. $\psi$. Noting that $\lim _{\psi \downarrow-\frac{1}{L-\Gamma_{00}(t)}} g_{1}(\psi ; t, \varphi)=+\infty$ and

$$
\lim _{\psi \uparrow+\infty} g_{1}(\psi ; t, \varphi)=\left[\left(\Gamma_{00}(t)-L\right) h_{1,00}(t)-\left(\Gamma_{01}(t)-\Gamma_{00}(t)\right) h_{I, 00}(t)\right] \varphi<0 .
$$

Apply the intermediate value theorem, and obtain a unique finite solution $\psi_{1}^{f o c}>-\frac{1}{L-\Gamma_{00}(t)}$ satisfying $g_{1}\left(\psi_{1}^{f o c} ; t, \varphi\right)=0$. Note that $\frac{\partial g_{1}(\psi ;, t, \varphi)}{\partial \psi}<0$ in its domain. Then, in light of Kumagai (1980)'s implicit function theorem, we also have that $\psi_{1}^{f o c}$ viewed as a function of $(t, \varphi)$ is $C^{1}$ in $(t, \varphi)$. For $g_{2}(\psi ; t, \varphi)$ and all $\psi$ satisfying $(33), g_{2}(\psi ; t, \varphi)$ is continuous and decreasing w.r.t. $\psi$. Note that $\lim _{\psi \downarrow-\frac{1}{L-\Gamma_{00}(t)}} g_{2}(\psi ; t, \varphi)=+\infty$, and

$$
\begin{aligned}
\lim _{\psi \uparrow+\infty} g_{2}(\psi ; t, \varphi)= & {\left[\left(\Gamma_{00}(t)-L\right) h_{1,00}(t)-\left(\Gamma_{01}(t)-\Gamma_{00}(t)\right) h_{I, 00}(t)\right] \varphi } \\
& -\Gamma_{00}(t)\left(r_{00}(t)-r\right) \varphi .
\end{aligned}
$$

Since $0<\Gamma_{00}(t)<\Gamma_{01}(t)<L$, and $r_{00}(t)-r>0$ for all $t \in[0, T]$ using Lemma 3, we have $\lim _{\psi \uparrow+\infty} g_{2}(\psi ; t, \varphi)<0$. Then applying the intermediate value theorem, there is a unique finite solution $\psi_{2}^{f o c}>-\frac{1}{L-\Gamma_{00}(t)}$ satisfying $g_{2}\left(\psi_{2}^{f o c} ; t, \varphi\right)=0$. Further, in light of Kumagai (1980)'s implicit function theorem, $\psi_{2}^{f o c}$, viewed as a function of $(t, \varphi)$ is $C^{1}$ in $(t, \varphi)$, since the derivative $\frac{\partial g_{2}(\psi ; t, \varphi)}{\partial \psi}<0$ in its domain.

Since $\Gamma_{00}(t)\left(r_{00}(t)-r\right) \varphi>0$ for all $(t, \varphi) \in[0, T] \times \mathbb{R}_{+}$, by (32) it follows immediately that for all $(t, \varphi) \in[0, T] \times \mathbb{R}_{+}, g_{2}(\psi ; t, \varphi)<g_{1}(\psi ; t, \varphi)$. This yields the validity of (36).

Proof of Lemma 6 Recall $g_{1}$ given in (32). Then, $\psi \rightarrow g_{1}(\psi ; t, \varphi)$ is continuous and decreasing on $\psi>-\frac{1}{L-\Gamma_{00}(t)}$. Moreover, we can decompose $g_{1}$ as $g_{1}(\psi ; t, \varphi)=$ $\bar{g}_{1}(\psi ; t, \varphi)+\hat{g}_{1}(\psi ; t, \varphi)$, where

$$
\begin{aligned}
\bar{g}_{1}(\psi ; t, \varphi):= & \left(\Gamma_{00}(t)-L\right) h_{1,00}(t) \varphi \\
& +\left[1+\psi\left(L-\Gamma_{00}(t)\right)\right]^{\gamma-1}\left(L-\Gamma_{00}(t)\right) h_{1,00}^{\mathbb{P}}(t) \varphi_{10}(t), \\
\hat{g}_{1}(\psi ; t, \varphi):= & -\left(\Gamma_{01}(t)-\Gamma_{00}(t)\right) h_{I, 00}(t) \varphi \\
& +\left[1+\psi\left(\Gamma_{01}(t)-\Gamma_{00}(t)\right)\right]^{\Gamma-1}\left(\Gamma_{01}(t)-\Gamma_{00}(t)\right) h_{I, 00}^{\mathbb{P}}(t) \varphi_{01}(t) .
\end{aligned}
$$

It is easy to check that $\bar{g}_{1}(\bar{\psi}(t, \varphi) ; t, \varphi)=\hat{g}_{1}(\hat{\psi}(t, \varphi) ; t, \varphi)=0$. Next, we prove that

$$
g_{1}(\max \{\bar{\psi}(t, \varphi), \hat{\psi}(t, \varphi)\} ; t, \varphi) \leq 0 .
$$

First we consider the case where $\bar{\psi}(t, \varphi) \leq \hat{\psi}(t, \varphi)$. In this case, $g_{1}(\hat{\psi}(t, \varphi) ; t, \varphi)=\bar{g}_{1}(\hat{\psi}(t, \varphi) ; t, \varphi)$, since $\hat{g}_{1}(\hat{\psi}(t, \varphi) ; t, \varphi)=0$. Note that $\psi \rightarrow$ $\bar{g}_{1}(\psi ; t, \varphi)$ is continuous and decreasing on $\psi>-\frac{1}{L-\Gamma_{00}(t)}$. Since $\bar{\psi}(t, \varphi) \leq$ 
$\hat{\psi}(t, \varphi), \bar{g}_{1}(\bar{\psi}(t, \varphi) ; t, \varphi) \geq \bar{g}_{1}(\hat{\psi}(t, \varphi) ; t, \varphi)$. Using $\bar{g}_{1}(\bar{\psi}(t, \varphi) ; t, \varphi)=0$, we obtain that

$$
g_{1}(\max \{\bar{\psi}(t, \varphi), \hat{\psi}(t, \varphi)\} ; t, \varphi)=\bar{g}_{1}(\hat{\psi}(t, \varphi) ; t, \varphi) \leq 0,
$$

yielding the inequality (57). By a symmetric argument, we can prove (57) when $\bar{\psi}(t, \varphi)>\hat{\psi}(t, \varphi)$, and omit the proof here. Since $g_{1}(\psi ; t, \varphi)$ is a continuous and decreasing function of $\psi>-\frac{1}{L-\Gamma_{00}(t)}, g_{1}\left(\psi_{1}^{f o c}(t, \varphi) ; t, \varphi\right)=0$. Using the inequality (36) in Lemma 5 , we then obtain the inequality (37).

Proof of Lemma 7 The limits (40) is obvious. Using the implicit function theorem and Lemma 6, it is not difficult to see that

$$
\frac{\psi_{1}^{f o c}(t, v)}{\partial v}=-\frac{\partial g_{1}\left(\psi_{1}^{f o c} ; t, v\right)}{\partial v} /\left.\frac{\partial g_{1}(\psi ; t, v)}{\partial \psi}\right|_{\psi=\psi_{1}^{f o c}}<0,
$$

since $0<\gamma<1$. Similarly, it also holds that

$$
\frac{\psi_{2}^{f o c}(t, v)}{\partial v}=-\frac{\partial g_{2}\left(\psi_{2}^{f o c} ; t, v\right)}{\partial v} /\left.\frac{\partial g_{2}(\psi ; t, v)}{\partial \psi}\right|_{\psi=\psi_{2}^{f o c}}<0 .
$$

This ends the proof.

Proof of Lemma 9 Combining Proposition 1, Lemma 6 and the estimate $0<$ $\Gamma_{00}(t)<\Gamma_{01}(t)<\delta L$ for some $\delta \in(0,1)$, it follows that there exist constants $K_{1}, \bar{K}_{1}>0$ so that

$$
\left|\psi^{*}(t, v)\right| \leq K_{1}+\bar{K}_{1} v^{\frac{1}{\gamma-1}}, \quad(t, v) \in[0, T] \times \mathbb{R}_{+} .
$$

Moreover, from Lemma 3 and (43), it follows that, for all $(t, v) \in[0, T] \times \mathbb{R}_{+}$,

$$
\begin{gathered}
|\bar{A}(t, v)| \leq \gamma\left\{r+\left(1+L\left|\psi^{*}(t, v)\right|\right)\left(h_{1,00}(0)+h_{I, 00}(0)\right)+2 L\left|\psi^{*}(t, v)\right|\left(\bar{m}_{1,00}+\bar{m}_{I, 00}\right)\right\} \\
+\bar{m}_{1,00}^{\mathbb{P}}+\bar{m}_{I, 00}^{\mathbb{P}} \leq K+\bar{K} v^{\frac{1}{\gamma-1}},
\end{gathered}
$$

for some constants $K, \bar{K}>0$. Similarly, using (39) and (58), there exist constants $K_{1}, K, \bar{K}>0$ so that

$$
\begin{aligned}
\bar{C}(t, v) & \leq \frac{1}{\gamma}\left[1+2 L\left|\psi^{*}(t, v)\right|\right]^{\gamma} \bar{m}_{1,00}^{\mathbb{P}}\left(1+\bar{m}_{I, 10}^{\mathbb{P}} T\right)+\frac{1}{\gamma}\left[1+2 L\left|\psi^{*}(t, v)\right|\right]^{\gamma} \bar{m}_{I, 00}^{\mathbb{P}} \\
& \leq K_{1}\left[1+2 L\left|\psi^{*}(t, v)\right|\right]^{\gamma} \leq K_{1}\left[1+\left(2 L\left|\psi^{*}(t, v)\right|\right)^{\gamma}\right] \\
& \leq K_{1}+K_{1}(2 L)^{\gamma}\left|\psi^{*}(t, v)\right|^{\gamma} \leq K+\bar{K} v^{\frac{\gamma}{\gamma-1}},
\end{aligned}
$$

where we use the inequality $(x+y)^{\gamma} \leq x^{\gamma}+y^{\gamma}$ for all $x, y \in \mathbb{R}_{+}$and $\gamma \in(0,1)$. This completes the proof of the lemma.

Proof of Lemma 10 Recall $A(t, \psi)$ and $C(t, \psi)$ defined in (43). It is immediately verified that $\psi \rightarrow A(t, \psi)$ is Lipschitz-continuous and $\psi \rightarrow C(t, \psi)$ is locally Lipschitz-continuous uniformly in time $t \in[0, T]$. Using Lemma $8, v \rightarrow \bar{A}(t, v)$ and $v \rightarrow \bar{C}(t, v)$ are locally Lipschitz-continuous uniformly in time $t \in[0, T]$. Since $v(t)$ is a solution to Eq. (46) by assumption, it is $C^{1}$ on $[0, T]$, and hence it 
is bounded on $[0, T]$. Then using the comparison theorem for first-order ODEs, we deduce that $v(t) \geq u(t)$ for all $t \in[0, T]$ using the inequality (44) and noting that $u(T)=a_{0} \leq \frac{1}{\gamma}=v(T)$.

Next, we prove that there exists a constant $\eta>0$ so that $u(t) \geq \eta$ for all $t \in[0, T]$. To this purpose, we first estimate the coefficient $A(t, \ell(t))$ and $C(t, \ell(t))$. Firstly, observe that $C(t, \ell(t))>0$ for all $t \in[0, T]$. On the other hand, note that

$$
0<\left(L-\Gamma_{00}(t)\right) h_{1,00}(t)+\left(\Gamma_{01}(t)-\Gamma_{00}(t)\right) h_{I, 00}(t)<L\left(\bar{m}_{1,00}+\bar{m}_{I, 00}\right),
$$

and noting that $\Gamma_{00}(t)$ on $t \in[0, T]$ is bounded, it follows that for all $t \in[0, T]$,

$$
\ell(t) \leq \frac{r+\bar{m}_{1,00}+\bar{m}_{I, 00}}{L \underline{m}_{1,00}-v} \times \frac{1}{1-e^{-\left(r+\bar{m}_{1,00}+\bar{m}_{I, 00}\right)\left(T_{1}-T\right)}}=: \delta_{T} .
$$

Then, using (43), it holds that for all $t \in[0, T]$,

$$
A\left(t, \psi^{0}(t)\right) \geq \gamma\left\{r-\delta_{T} L\left(\bar{m}_{1,00}+\bar{m}_{I, 00}\right)\right\}-\bar{m}_{1,00}^{\mathbb{P}}-\bar{m}_{I, 00}^{\mathbb{P}}=: \eta_{0} .
$$

Recall Eq. (45). The solution $(u(t) ; t \in[0, T])$ admits the following form

$$
\begin{gathered}
u(t)=a_{0} e^{\int_{t}^{T} A\left(s, \psi^{0}(s)\right) d s}+\int_{t}^{T} C\left(s, \psi^{0}(s)\right) e^{\int_{t}^{s} A\left(\iota, \psi^{0}(\iota)\right) d} d s \\
\geq a_{0} e^{\int_{t}^{T} A\left(s, \psi^{0}(s)\right) d s} \geq a_{0} e^{\eta_{0}(T-t)} .
\end{gathered}
$$

Define the constant given by

$$
\eta:=\left\{\begin{array}{cl}
a_{0}, & \text { if } \eta_{0} \geq 0 \\
a_{0} e^{\eta_{0} T}, & \text { if } \eta_{0}<0 .
\end{array}\right.
$$

Then, it holds that $u(t) \geq \eta$ for all $t \in[0, T]$. This implies that $v(t) \geq u(t) \geq \eta$, for all $t \in[0, T]$.

Acknowledgements The author gratefully acknowledges the constructive and insightful comments and suggestions provided by Prof. Agostino Capponi, Prof. Stéphane Crépey two anonymous reviewers, which contributed to improve the quality of the manuscript greatly. The research was partially supported by NSF of China (No. 11471254), The Key Research Program of Frontier Sciences, CAS (No. QYZDB-SSWSYS009) and Fundamental Research Funds for the Central Universities (No. WK3470000008).

\section{Competing interests}

The authors declare that they have no competing interests.

\section{References}

Azizpour, S, Giesecke, K, Schwenkler, G: Exploring the sources of default clustering. J. Finan. Econom. Forthcoming (2017)

Belanger, A, Shreve, S, Wong, D: A general framework for pricing credit risk. Math. Finan 14, 317-350 (2004)

Bielecki, T, Jang, I: Portfolio optimization with a defaultable security. Asia-Pacific Finan. Markets 13, 113-127 (2006)

Bielecki, T, Jeanblanc, M, Rutkowski, M: Pricing and trading credit default swaps in a hazard process model. Ann. Appl. Probab 18, 2495-2529 (2008) 
Bielecki, T, Rutkowski, M: Valuation and hedging of contracts with funding costs and collateralization. SIAM J. Finan. Math 6, 594-655 (2015)

Bloomberg News, November 8, 2013: Report available at http://www.bloomberg.com/news/2013-11-08/ pimco-said-to-wager-10-billion-in-default-swaps-credit-markets.html

Bo, L, Wang, Y, Yang, X: An optimal portfolio problem in a defaultable market. Adv. Appl. Probab 42, 689-705 (2010)

Bo, L, Capponi, A: Optimal investment in credit derivatives portfolio under contagion risk. Math. Finan 26, 785-834 (2016)

Bo, L, Capponi, A: Optimal credit investment with borrowing costs. Math Oper. Res 42, 546-575 (2017)

Capponi, A, Figueroa-López, JE: Dynamics portfolio optimization with a defaultable security and regimeswitching. Math Finan 24, 207-249 (2014)

Chen, H: Macroeconomic conditions and the puzzles of credit spreads and capital structure. J. Finan $\mathbf{6 5}$, 2171-2212 (2010)

Crépey, S: Bilateral counterparty risk under funding constraints. Part I: pricing. Math Finan 25, 23-50 (2015)

Cvitanić, J, Karatzas, I: Hedging contingent claims with constrained portfolios. Ann. Appl. Probab 3, 652-681 (1993)

Draouil, O, Oksendal, B: A donsker delta functional approach to optimal insider control and applications to finance. Commun. Math Stats 3, 365-421 (2015)

Duffie, D, Singleton, K: Credit Risk. Princeton University Press, Princeton (2003)

El Karoui, N, Peng, S, Quenez, MC: Backward stochastic differential equations in finance. Math Finan 7, $1-71(1997)$

Frey, R, Backhaus, J: Pricing and hedging of portfolio credit derivatives with interacting default intensities. Int. J. Theor. Appl. Finan 11, 611-634 (2008)

Giesecke, K, Kim, B, Kim, J, Tsoukalas, G: Optimal credit swap portfolios. Manage Sci 60, 2291-2307 (2014)

ISDA News Release: ISDA Announces Successful Implementation of Big Bang' CDS Protocol; Determinations Committees and Auction Settlement Changes Take Effect (2009). Available at http://www. isda.org/press/press040809.html

Jarrow, R, Yu, F: Counterparty risk and the pricing of defaultable securities. J. Finan 56, 1765-1799 (2001)

Jiao, Y, Pham, H: Optimal investment with counterparty risk: a default density approach. Finan. Stoch 15, 725-753 (2011)

Korn, R: Contingent claim valuation in a market with different interest rates. Math. Meth. Oper. Res 42, 255-274 (1995)

Korn, R, Kraft, H: Optimal portfolios with defaultable securities-a firm value approach. Int. J. Theor. Appl. Finan 6, 793-819 (2003)

Kraft, H, Steffensen, M: Portfolio problems stopping at first hitting time with application to default risk. Math. Meth. Oper. Res 63, 123-150 (2005)

Kumagai, S: An implicit function theorem: comment. J. Optim. Theor. Appl 31, 285-288 (1980)

Mercurio, F: Bergman, Piterbarg, and Beyond: pricing derivatives under collateralization and differential rates. In: Londoño, J, Garrido, J, Hernández-Hernández, D (eds.) Actuarial Sciences and Quantitative Finance. Springer Proceedings in Mathematics \& Statistics, vol 135. Springer, Cham (2015) 